EPJ Web of Conferences 60, 17006 (2013)

DOI: $10.1051 /$ epjconf $/ 20136017006$

(C) Owned by the authors, published by EDP Sciences, 2013

\title{
Searches for Long-Lived Particles with the CMS experiment
}

\author{
Livia Soffi ${ }^{1,2, a}$ \\ ${ }^{1}$ Sapienza, Universita' di Roma \\ ${ }^{2}$ INFN, Sez. di Roma
}

\begin{abstract}
We present searches for long-lived particles at CMS. These include long-lived gluinos, fractionally and multiply charged particles, and displaced particles that decay into leptons, photons, or jets.
\end{abstract}

\section{Introduction}

The Standard Model (SM) of elementary particles and their fundamental interactions has been verified with high accuracy by several experiments, showing an excellent agreement between theoretical expectations and experimental results. Despite the striking experimental success, this theory still has some drawbacks and unsolved problems, that range from the origin of particle masses to the nature of the Dark Matter in the Universe and represent a strong conceptual indication for physics in the TeV energy domain.

Recent theoretical and experimental advances have brought a new focus on theoretical proposals for physics beyond the Standard Model (BSM) with massive and longliving particles, which are common in several Supersymmetry (SUSY) models and also in more exotic scenarios. From an experimental point of view, models with longlived particles are very accredited for early discoveries of new physics from the experiments at the Large Hadron Collider (LHC), the proton-proton collider at the CERN laboratories of Geneva. The major advantage of many of these models consists in non negligible cross sections and relatively small SM background.

Searches for heavy stable charged particles and longliving neutralinos performed with the Compact Muon Solenoid (CMS) detector [1] are here presented and interpreted in terms of two main theoretical models, gauge mediated supersymmetry breaking (GMSB) and split supersymmetry. In GMSB [2], the gravitino is usually the lightest supersymmetric particle (LSP), so the Next-LSP is naturally long-lived, as it must decay gravitationally . In split supersymmetry [3] gluinos, which can decay only via virtual squarks to the lightest SUSY particle, has a long lifetime, which can vary from microseconds to $10^{10}$ years depending on its mass and the value of the SUSY-breaking scale.

The data used here were collected by CMS detector in 2011 and in 2012 using proton-proton collisions at a center of mass energy of 7 and $8 \mathrm{TeV}$ at the LHC.

\footnotetext{
a-mail: livia.soffi@cern.ch
}

\section{Heavy Stable Charged Particles}

Many extensions of the Standard Model include heavy ( $m>100 \mathrm{GeV}$ ), long-lived, charged particles [4] that have speed, v, less than the speed of light, $c$, and/or charge, $Q$, not equal to $\pm 1 e$. With lifetimes greater than a few nanoseconds, these particles can travel distances larger than the typical collider detector and appear stable (in analogy to the pion or kaon). These particles can be generically referred to as heavy stable charged particles (HSCPs) and can be singly charged $(|Q|=1 e)$, fractionally charged $(|Q|<1 e)$, or multiply charged $(|Q|>1 e)$.

Typically, HSCPs are slower $(\beta<c)$ than SM particles produced at the same p-p collision and are detected with a measurable time delay inside the detector and with an anomalous ionization energy loss $(d E / d x)$ in the inner tracker system. The information about the particle velocity combined with the standard momentum measurement allows to determine the HSCP mass based on the relationship shown in eq. 1:

$$
I_{h}=K \frac{m^{2}}{p^{2}}+C
$$

where $I_{h}$ is an estimator of $d E / d x$ and the empirical parameters $\mathrm{K}$ and $\mathrm{C}$ are determined from data using a sample of low-momentum protons.

HSCPs can be categorized into two types: lepton-like or hadron-like. Lepton-like HSCPs such as the stau, behave like heavy muons and interact primarily through the electromagnetic force while hadron-like HSCPs such as gluino and stop, since they are coloured, additionally interact through the strong force and form bound states with SM quarks (or gluons) called R-hadrons. R-hadrons can be charged or neutral. Strong interactions between the SM quarks and detector material increase energy loss and can lead to charge exchange, e.g. converting charged Rhadrons into neutral ones (and vice-versa).

The analyses of HSCP candidates fall into multiple topologies. Singly charged HSCPs are searched for in three ways: (1) requiring tracks reconstructed in both the inner silicon detectors and the muon system, referred to 
as the tracker+TOF analysis; (2) only requiring tracks reconstructed in the inner silicon detectors, the trackeronly analysis; and (3) only requiring tracks reconstructed in the muon system, the muon-only analysis. The latter two cases account for the possibility of charge flipping (charged to neutral or vice versa) within the calorimeter.

Fig. 1 shows $I_{h}$ versus momentum for tracks from data and simulated HSCP signals with various charges. In the momentum range of interest at the LHC (10-1000 GeV), SM particles interact with nearly flat ionization energy loss $(\sim 3 \mathrm{MeV} / \mathrm{cm})$. Searching for candidates with larger $d E / d x$ gives sensitivity to massive particles with $|Q|=1 e$, particles with $|Q|>1 e$, and low momentum particles with $|Q|<1 e$. On the other hand, searching for candidates with lower $d E / d x$ yields sensitivity to high momentum particles with $|Q|<1 e$.

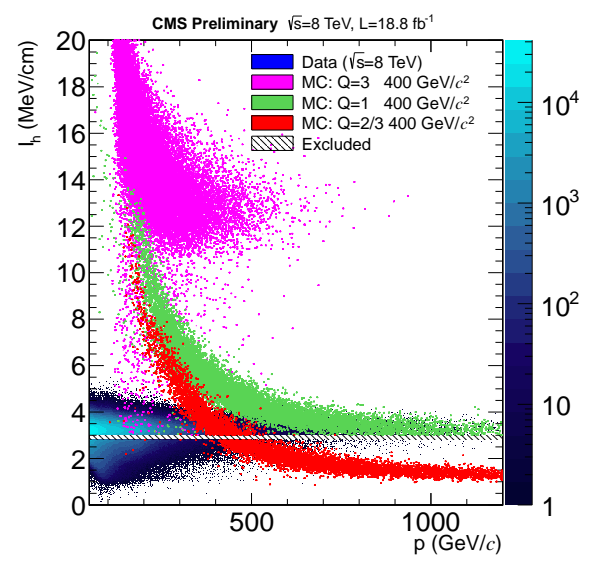

Figure 1. Distribution of $I_{h}$ versus particle momentum for data and singly, fractionally, and multiply charged HSCP candidates.

The time of flight to the muon system can also be used to discriminate between speed-of-light particles and slower candidates. A single $\delta t$ measurement can be used to determine the track $\beta^{-1}$ via the equation:

$$
\beta^{-1}=1+\frac{c \delta t}{L}
$$

where $\mathrm{L}$ is the flight distance.

Previous collider searches for HSCPs have been performed at LEP [5], HERA [6], the Tevatron [7], and the LHC [8]. The results from such searches have placed important bounds on BSM theories such as lower limits at 95\% confidence level on the mass of gluinos, scalar top quarks and pair produced scalar tau at 1098, 737 and 223 $\mathrm{GeV}$, respectively. Presented here are several searches for singly, fractionally, and multiply charged HSCPs in data collected with the CMS detector for the complete 2011 $\left(\sqrt{s}=7 \mathrm{TeV}, 5.0 \mathrm{fb}^{-1}\right)$ and $2012(\sqrt{s}=8 \mathrm{TeV}, 18.8$ $\left.f b^{-1}\right)$ runs.

\subsection{Selection and results}

High transverse momentum $\left(P_{T}\right)$ muon and missing transverse energy $\left(B_{T}\right)$ triggers are used to collect collision data [9]. As the instantaneous LHC luminosity increased, the muon $P_{T}$ threshold had to be increased from 30 to $45 \mathrm{GeV}$. The missing transverse energy trigger was at a threshold of $150 \mathrm{GeV}$ throughout the run, based on the particle flow reconstruction [10]. The $\phi_{T}$ trigger allows for recovery of some events in which no R-hadrons is charged in both the inner tracker and muon subsystems. The particle-flow algorithm rejects tracks reconstructed only in the inner tracker with a track $P_{T}$ much greater than the matched energy deposited in the calorimeter as would be the case for HSCPs becoming neutral in the calorimeter. The algorithm also rejects tracks reconstructed only in the muon system. Thus only the energy that these HSCPs deposit in the calorimeter, roughly $10-20 \mathrm{GeV}$, will be included in the $H_{T}$ calculation. In events in which no HSCPs are reconstructed as muon candidates, significant $H_{T}$ will result if the vector sum of the HSCPs' momenta is large.

The selection criteria exploit the transverse momentum, the energy loss and the time of flight of these particles.

For all the analyses, the results are based on a counting experiment in the final selection with a data driven method used to estimate the background contribution to the signal region. Candidates passing the preselection are then subject to two (or three) additional criteria based on $P_{T}, d E / d x$ and TOF to further improve the signal to background ratio. By choosing criteria that are uncorrelated, it is possible to use the candidates that fail one (or more) of these criteria to predict the background with the ABCD method (where $\mathrm{D}$, the background expectation in the signal region, is estimated by $\mathrm{D}=\mathrm{BC} / \mathrm{A}$, where $\mathrm{B}(\mathrm{C})$ is the number of candidates that fail the first (second) criteria but pass the other one, and $\mathrm{A}$ is the number of candidates that fail both criteria).

Observed and predicted mass spectra, computed using eq. 1, are shown in fig. 2 for the tracker-only 2011-2012 combined analysis. The expected distribution for a representative signal is shown in green. Events are counted in the mass window between the nominal HSCP mass minus two sigma and $2 \mathrm{TeV}$, where sigma is the expected mass resolution.

No significant excess of events is observed over the predicted background. Cross section limits are placed at 95\% CL for both 7 and $8 \mathrm{TeV}$ using a $C L_{s}$ approach [11]. Figure 3 shows the limits as a function of mass for the tracker + TOF 2011-2012 combined analysis.

\section{New physics with displaced photons}

The version of supersymmetry based on gauge-mediated SUSY breaking is of particular theoretical interest for physics beyond the SM as it not only stabilizes the mass of the SM Higgs boson, but also avoids the large flavorchanging neutral currents that trouble other SUSY- breaking scenarios [2]. According to a GMSB benchmark scenario, commonly described as Snowmass Points and Slopes 8 (SPS8) [12], the gravitino $(\tilde{G})$ is the LSP and the lightest neutralino $\left(\tilde{\chi}_{1}^{0}\right)$ is the next-to-lowest mass SUSY particle (NLSP) and decays predominantly into a gravitino 


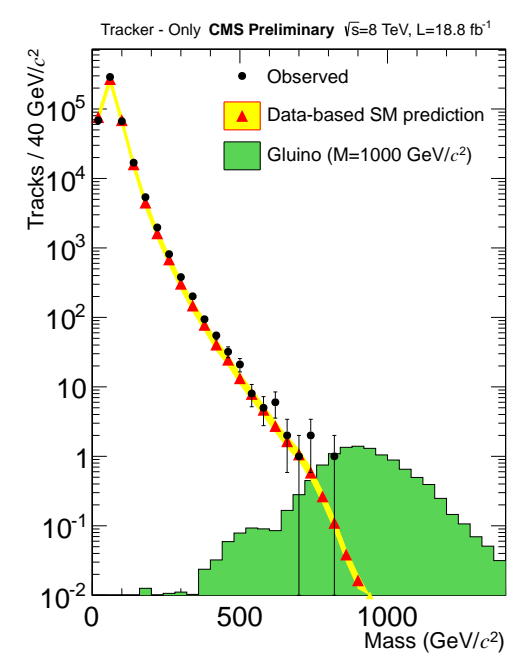

Figure 2. Observed and predicted mass spectra. The expected distribution for a representative signal is shown in green.

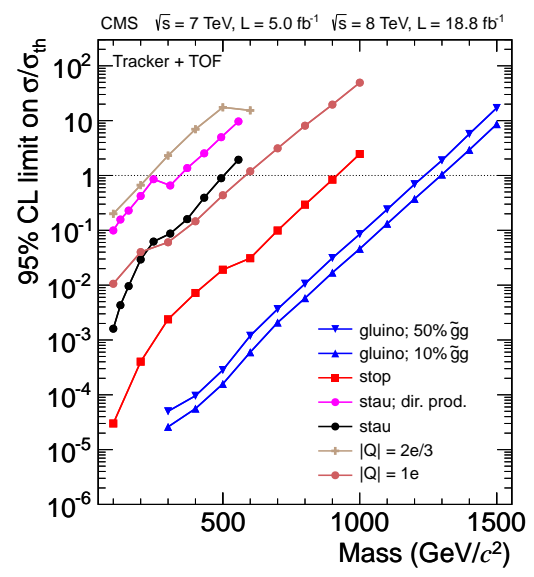

Figure 3. Cross section upper limits at $95 \%$ C.L. on various signal models for the tracker+TOF analysis. In the legend, CS stands for charged suppressed interaction mode.

and a photon. Figure 4 shows a diagram of possible squark and gluino pair-production process that result in a singlephoton final state.

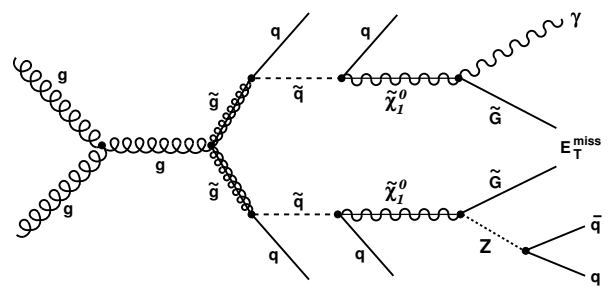

Figure 4. Example diagram for SUSY process that result in a single-photon final state through gluino production at the LHC.

The combination of energetic photons, the significant energy imbalance in the transverse plane (due to gravitino which escapes from detection) and the numerous high $P_{T}$ jets from the many quarks produced in the decay chains, generally leads to extremely clean, nearly background-free analysis.

Even more interesting, from an experimental point of view, is the case of a long-lived neutralino. If the lifetime is not too large, the large majority of $\tilde{\chi}_{1}^{0}$ travel through the detector and finally decay at a certain place which is displaced from the interaction point. In addition to this, a photon from a long-lived $\tilde{\chi}_{1}^{0}$ is typically out-of-time with respect to a photon from a promptly decay.

The excellent performance of the electromagnetic calorimeter (ECAL) allows also for the identification of off-pointing and off-time photons from the decay of longlived particles, thanks to the optimal resolution $(<1 \mathrm{~ns}$ for $E>3 \mathrm{GeV}$ [13]) on time measurement. The time of arrival of the photon in ECAL will be referred as ECAL Time. Figure 5 shows a comparison between the distributions of ECAL Time for photons from SM events, and photons from long-living neutralino with different $\tilde{\chi}_{1}^{0}$ lifetimes. All distributions have been normalized to unity. The plot clearly demonstrates that GMSB distribution populates the region at large reconstructed time and that the measured time in ECAL is a very powerful variable in identifying out-of-time photons. In this analysis signal is expected when both large $B_{T}$ and ECAL Time are measured.

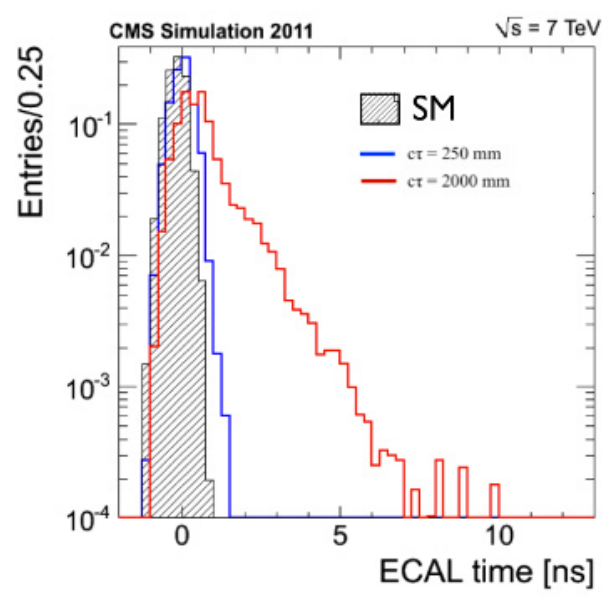

Figure 5. ECAL time distribution for prompt and off-time photons. Two different GMSB scenario are shown with neutralino proper decay length equal to $250 \mathrm{~mm}$ and $2000 \mathrm{~mm}$.

Previous searches for long-lived neutralinos have been performed by the CMS Collaboration [14], using the impact parameter of converted photons relative to the beam collision point, and by the CDF [15] collaboration, using only the missing transverse energy in the event. Other searches with prompt photons by the ATLAS [16] and D0 [17] collaborations, place lower limits on the mass of the $\tilde{\chi}_{1}^{0}$ at $280 \mathrm{GeV}$ and $175 \mathrm{GeV}$, respectively, in the SPS 8 scenario. 


\subsection{Objects reconstruction}

The photon candidates are reconstructed from clusters of energy in the ECAL. The ECAL was designed to have both excellent energy resolution and high granularity, making it a powerful instrument to measure photons with high precision at the LHC. Photon objects are created based on depositions of energy within the ECAL (supercluster). A supercluster passing a list of identification and quality criteria (e.g. shower shape) is promoted to a photon as described in [18]. The absence of pixel tracker hits matching to the supercluster is required to discriminate photons from electrons. To increase the purity of the photon sample, isolation requirements are applied using combined information from ECAL, HCAL and the tracker. Jets are clustered with the anti-kT clustering algorithm [19] with a size parameter $\mathrm{R}=0.5$ and reconstructed with the Particle-Flow reconstruction algorithm [10]. Within the Particle-Flow framework the $Z_{T}$ is the magnitude of the negative vector sum of all reconstructed particles.

\subsection{Selection and results}

This analysis [20] selects one high $P_{T}$ isolated photon with at least three jets in the final state. The trigger threshold on photon $P_{T}$ has evolved during the data taking from 75 $\mathrm{GeV}$ to $90 \mathrm{GeV}$, adding in the end the requirement of three jets at least, with $P_{T}$ above $25 \mathrm{GeV}$.

Irreducible SM backgrounds, i.e. events with real missing transverse energy and high $P_{T}$ photons in the final state, are negligible. The main backgrounds are instead reducible and arise from SM processes with misidentified photons and/or mis-measured missing transverse energy like QCD events and $\gamma+$ jets. The contribution of these two main backgrounds are estimated to make up more than $99 \%$ of the total data sample and are estimated using datadriven control samples. The remaining $1 \%$ are other (nonQCD) processes taken into account since they can play a role in the tails of the $Z_{T}$ distribution where signal is expected. Simulated events are used to estimate the contribution of these processes.

To estimate the number of background and signal events in data, a maximum likelihood fit is performed to the two-dimensional distribution of ${\#_{T}}_{T}$ and ECAL Time. The one-dimensional projections of $H_{T}$ and ECAL Time for the data and expected backgrounds, as determined from the fit, are illustrated in fig. 6 .

The observed event yield in data is consistent with the SM background prediction, and upper limits are obtained on the production cross section of a long-lived neutralino in the context of the GMSB model, assuming that $\mathcal{B}\left(\tilde{\chi}_{1}^{0} \rightarrow \gamma+\tilde{G}\right)=100 \%$.

Exclusion limits are provided in the mass and proper decay length plane of the long-lived $\tilde{\chi}_{1}^{0}$ in fig. 7.

\section{Conclusion}

The CMS experiment has performed searches for longlived particles using LHC proton-proton collision data at a center-of-mass energy of 7 and $8 \mathrm{TeV}$.
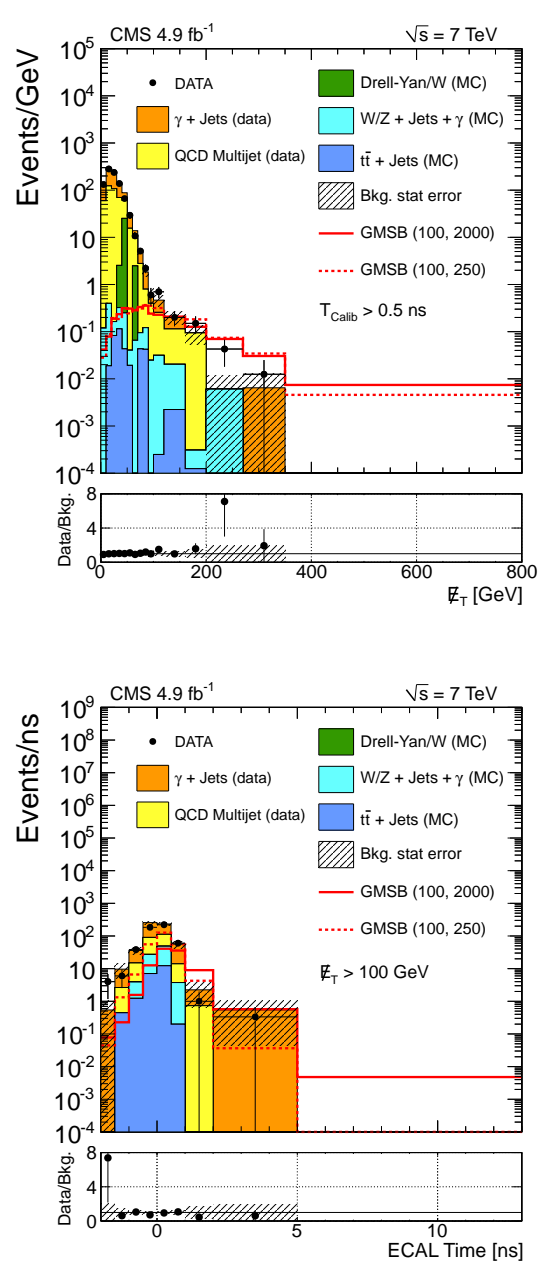

Figure 6. The one-dimensional projection after all selection requirements for $B_{T}$ for events with ECAL Time $>0.5 \mathrm{~ns}$ and for ECAL timing for events with $B_{T}>100 \mathrm{GeV}$. The multijet and $\gamma+$ jets backgrounds are normalized to the yields from the fit. The rest of the backgrounds are fixed according to the integrated luminosity of the data. The GMSB $(100,2000)$ benchmark point corresponds to $\Lambda=100 \mathrm{TeV}, c \tau=2000 \mathrm{~mm}$ and the GMSB $(100,250)$ benchmark point corresponds to $\Lambda=100 \mathrm{TeV}$, $c \tau=250 \mathrm{~mm}$.

Mass limits for gluinos, stops, staus, fractionally charged particles, and multiply charged particles ranging up to $1322 \mathrm{GeV}$ for gluinos, are the most restrictive to date eclipsing previous limits from the LHC.

A search for Long-lived neutralino decaying to a photon and a gravitino is performed using the missing transverse energy and the timing information from the ECAL. This is the first CMS analysis that exploit the time measured by the ECAL. No significant excess of events beyond the SM contributions has been observed, and upper limits at $95 \% \mathrm{CL}$ are obtained on the GMSB production cross section in the SPS8 model of GMSB supersymmetry. The mass of the lightest neutralino is then restricted to values $\mathrm{m}\left(\tilde{\chi}_{1}^{0}\right)>220 \mathrm{GeV}$ (for neutralino proper decay length $c \tau<500 \mathrm{~mm}$ ) and the neutralino decay length $c \tau$ must be greater than $6000 \mathrm{~mm}$ (for $\mathrm{m}\left(\tilde{\chi}_{1}^{0}\right)<150 \mathrm{GeV}$ ). 


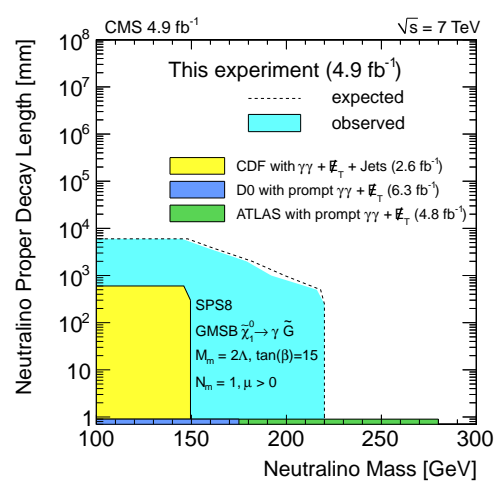

Figure 7. The observed exclusion region for the mass and proper decay length of the $\tilde{\chi}_{1}^{0}$ in the SPS8 model of GMSB supersymmetry.

These limits are the most stringent for long-lived neutralinos.

\section{References}

[1] CMS Collaboration, The CMS experiment at the CERN LHC JINST 3:S08004, (2008).

[2] G.F. Giudice and R. Rattazzi, Theories with gaugemediated supersymmetry breaking, Physics Reports 322, 419-499 (1999), doi:10.1016/S03701573(99)00042-3.

[3] N. Arkani-Hamed and S. Dimopoulos and G.F. Giudice and A. Romanino, Aspects of Split Supersymmetry, Nuclear Physics B 709, 3-46 (2005), doi:10.1016/j.nuclphysb.2004.12.026.

[4] M. Drees and X. Tata, Signals for heavy exotics at hadron colliders and supercolliders, Phys. Lett. B 252, 695 (1990), doi:10.1016/0370-2693(90)90508-4.

[5] ALEPH Collaboration, Search for pair production of long-lived heavy charged particles in $e^{+} e^{-}$annihilation, Phys. Lett. B 405, 379-388 (1997), doi:10.1016/S0370-2693(97)00715-6.

[6] H1 Collaboration, Measurement of anti-deuteron photoproduction and a search for heavy stable charged particles at HERA, Eur. Phys. J. C 36, 41323 (2004), doi:10.1140/epjc/s2004-01894-1.

[7] CDF Collaboration, Search for long-lived massive charged particles in $1.96 \mathrm{TeV} p \bar{p}$ collisions, Phys. Rev. Lett. 103, (2009) 021802, doi:10.1103/PhysRevLett.103.021802.
[8] ATLAS Collaboration, Search for heavy long-lived charged particles with the ATLAS detector in pp collisions at $\sqrt{s}=7 \mathrm{TeV}$, Phys. Lett. B 703, 428-446 (2011), doi:10.1016/j.physletb.2011.08.042.

[9] CMS Collaboration, Search for heavy long-lived charged particles in pp collisions at $\sqrt{s}=7$ and 8 $T e V$, arXiv:1305.0491.

[10] CMS Collaboration, Jet performance in pp collisions at Analysis Summary, CMS-PAS-JME-10-003 (2010).

[11] A. L. Read, Modified frequentist analysis of search results (The $C L(s)$ method).

[12] B. C. Allanach et al., The Snowmass Points and Slopes: benchmarks for SUSY searches, Eur. Phys. J. C 25, 113 (2002), doi:10.1007/s10052-002-0949-3.

[13] CMS Collaboration, Time reconstruction and performance of the CMS electromagnetic calorimeter, JINST 5 (2010) T03011, doi:10.1088/17480221/5/03/T03011.

[14] CMS Collaboration, Search for new physics with long-lived particles decaying to photons and missing energy in pp collisions at $\sqrt{s}=7 \mathrm{TeV}$, arXiv:1207.0627,

[15] CDF Collaboration, Search for Supersymmetry with Gauge-Mediated Breaking in Diphoton Events with Missing Transverse Energy at CDF II, Phys. Rev. Lett. 104, (2010) 011801, doi:10.1103/PhysRevLett.104.011801.

[16] ATLAS Collaboration, Search for diphoton events with large missing transverse momentum in 7 $\mathrm{TeV}$ proton-proton collision data with the ATLAS detector, Phys. Lett. B 710, (2012) 519, doi:10.1016/j.physletb.2012.02.054.

[17] D0 Collaboration, Search for diphoton events with large missing transverse energy in $6.3 \mathrm{fb}^{-1}$ of $p \bar{p}$ collisions at $\sqrt{s}=1.96 \mathrm{TeV}$, Phys. Rev. Lett. 105, (2010) 221802, doi:10.1103/PhysRevLett.105.221802.

[18] CMS Collaboration, Photon reconstruction and identification at $\sqrt{(} s)=7 \mathrm{TeV}$, CMS Physics Analysis Summary CMS-PAS-EGM-10-005, (2010).

[19] M. Cacciari, G. Salam, and G. Soyez, The anti-kt jet clustering algorithm, Journal of High Energy Physics 04 (2008), doi:10.1088/1126-6708/2008/04/063.

[20] CMS Collaboration, Search for long-lived particles in events with photons and missing energy in proton-proton collisions at $\sqrt{s}=7$ $\mathrm{TeV}$, Physics Letters B 722, 273-294 (2013), doi:10.1016/j.physletb.2013.04.027. 